\title{
Two-Year Outcomes of Visian Implantable Collamer Lens with a Central Hole for Correcting High Myopia
}

\author{
Zhipeng Yan $\mathbb{D}^{1}{ }^{1}$ Huamao Miao, ${ }^{2,3,4}$ Feng Zhao, ${ }^{2,3,4}$ Xiaoying Wang $\mathbb{D}^{2,3,4}$ Xun Chen, ${ }^{2,3,4}$ \\ Meiyan Li, ${ }^{2,3,4}$ and Xingtao Zhou iD ${ }^{2,3,4}$ \\ ${ }^{1}$ Department of Ophthalmology, Third Hospital of Hebei Medical University, Shijiazhuang, Hebei, China \\ ${ }^{2}$ Department of Ophthalmology and Optometry, Eye and ENT Hospital, Fudan University, Shanghai, China \\ ${ }^{3}$ NHC Key Laboratory of Myopia, Fudan University, Shanghai, China \\ ${ }^{4}$ Shanghai Research Center of Ophthalmology and Optometry, Shanghai, China
}

Correspondence should be addressed to Xingtao Zhou; doctzhouxingtao@163.com

Received 4 February 2018; Revised 21 May 2018; Accepted 30 May 2018; Published 3 July 2018

Academic Editor: Tamer A. Macky

Copyright (C) 2018 Zhipeng Yan et al. This is an open access article distributed under the Creative Commons Attribution License, which permits unrestricted use, distribution, and reproduction in any medium, provided the original work is properly cited.

Purpose. To investigate two-year outcomes of Visian Implantable Collamer Lens with a central hole (ICL V4c) implantation for correcting high myopia. Methods. Sixty-one eyes of 32 patients went through ICL V4c implantation. Safety, efficacy, predictability, and intraocular pressure were evaluated 2 years postoperatively. Anterior chamber volume (ACV), anterior chamber depth (ACD), anterior chamber angle width (ACAW), and vault were measured using a Scheimpflug tomography imaging system. Results. At 2 years, the spherical equivalent refraction decreased from preoperative $-14.62 \pm 4.29 \mathrm{D}$ to $-0.90 \pm 0.95 \mathrm{D}$, with $79 \%$ of the eyes within $\pm 0.50 \mathrm{D}$ and $98 \%$ within $\pm 1.00 \mathrm{D}$ of the intended correction. The efficacy index was $1.03 \pm 0.23$, and the safety index was $1.24 \pm 0.26$. Corrected distance visual acuity (CDVA) remained unchanged in $23 \%$ of the eyes, $75 \%$ gained one or more lines of CDVA, and $2 \%$ lost one line. The ACV, ACD, and ACAW, respectively, decreased from $193.28 \pm 29.15 \mathrm{~mm}^{3}, 3.15 \pm 0.23 \mathrm{~mm}$, and $36.51 \pm 6.54$ degree to $112.48 \pm 17.01 \mathrm{~mm}^{3}, 2.99 \pm 0.23 \mathrm{~mm}$, and $22.54 \pm 5.27$ degree $(p=0.0008,0.008$, and 0.0003 , resp.). Intraocular pressure was $15.39 \pm 2.88 \mathrm{mmHg}$ before surgery and was $15.86 \pm 4.11 \mathrm{mmHg}$ at 2 years $(p=0.11)$. Conclusion. Implantation of ICL V4c is a safe, effective, and predictable procedure for correcting high myopia. Reduction of anterior chamber space after surgery did not induce intraocular pressure increase during the 2-year follow-up.

\section{Introduction}

The high prevalence of high myopia in Chinese young adults has become a social public health issue in China [1]. Currently, laser refractive surgery remains the most common surgical procedure to correct myopia. However, it has limitations in correcting high myopia. The posterior chamber phakic intraocular lens implantation could correct a wider range of myopia, and it has become an important surgical option for patients who are unsuitable for corneal refractive surgeries.

The Visian Implantable Collamer Lens V4c (ICL V4c) is a new generation of posterior chamber phakic intraocular lens, which is capable to correct up to $-18.00 \mathrm{D}$ refraction [2]. Compared with the traditional ICL V4, the ICL V4c was designed with a $0.36 \mathrm{~mm}$ central hole, which improves aqueous humor circulation and obviates the need for peripheral iridotomy or iridectomy. To fully understand the safety of this technique, the present 2-year study evaluated refractive outcomes, anterior segment changes, and lens position after ICL V4c implantation in patients with myopia of $-8.00 \mathrm{D}$ or higher.

\section{Patients and Methods}

This is a nonrandomized prospective study. All the patients underwent routine ophthalmic examinations at the Refractive Surgery Center of the Department of Ophthalmology, Eye and ENT Hospital of Fudan University (Shanghai, People's Republic of China), and met the surgical requirements for ICL V4c (STAAR Surgical Company, Monrovia, California, USA) implantation. The inclusion 
TABle 1: Patient demographic data and ICL V4c characteristics.

\begin{tabular}{lcc}
\hline Parameters & Mean \pm SD & Range (min, max) \\
\hline Age (year) & $30.87 \pm 8.03$ & $20.00,45.00$ \\
Spherical equivalent (D) & $-14.62 \pm 4.29$ & $-25.75,-8.00$ \\
Refractive cylinder (D) & $-1.82 \pm 1.22$ & $-5.75,0.00$ \\
CDVA (decimal) & $0.83 \pm 0.24$ & $0.20,1.20$ \\
ICL V4c size (mm) & $12.93 \pm 0.42$ & $12.10,13.70$ \\
ICL V4c power (D) & $-14.10 \pm 2.85$ & $-18.00,-8.00$ \\
\hline
\end{tabular}

ICL V4c=Visian Implantable Collamer Lens with a central hole; $\mathrm{SD}=$ standard deviation; $\mathrm{D}=$ diopters $; \mathrm{CDVA}=$ corrected distance visual acuity; $\mathrm{mm}=$ millimeter.

criterion was with preoperative spherical equivalent (SE) of $-8.00 \mathrm{D}$ or higher. Exclusion criteria were corneal endothelial cell density (ECD, noncontact specular microscopy, SP-2000P, Topcon Corporation, Japan) less than $2000 \mathrm{cell} / \mathrm{mm}^{2}$, a history of ocular surgery, cataract, glaucoma, amblyopia, retinal detachment, neuroophthalmic diseases, and ocular inflammatory diseases. Thirty-two patients (61 eyes) with 14 males and 18 females were consecutively enrolled, and none was lost at two years. Patient demographic data and ICL V4c characteristics are listed in Table 1. Their mean age was $30.87 \pm 8.03$ years (range, 20 to 45 years). The mean preoperative SE was $-14.62 \pm 4.29 \mathrm{D}$ (range, $-8.00 \mathrm{D}$ to $-25.75 \mathrm{D}$ ), and 23\% (14 eyes) of the eyes had preoperative SE over $-18.00 \mathrm{D}$.

The study adhered to the tenets of the Declaration of Helsinki and was approved by the Ethics Committee of the Eye and ENT Hospital, Fudan University. All patients signed their informed consent after a detailed explanation of the possible risks and benefits of the study.

\section{Surgical Technique}

The ICL V4c is a plate-haptic single-piece intraocular lens made of Collamer. It has a central convex-concave optical zone and incorporates a forward vault to minimize contact with the crystalline lens. A $360 \mu \mathrm{m}$ central hole was included to improve aqueous humor circulation, which eliminates the need for preoperative laser peripheral iridotomy. The ICL $\mathrm{V} 4 \mathrm{c}$ corrects $-0.50 \mathrm{D}$ to $-18.00 \mathrm{D}$ myopic spherical refraction and up to $-5.00 \mathrm{D}$ cylindrical refraction. There are 4 sizes: $12.1 \mathrm{~mm}, 12.6 \mathrm{~mm}, 13.2 \mathrm{~mm}$, and $13.7 \mathrm{~mm}$. Power calculation of the ICL V4c was performed by the manufacturer (STAAR Surgical) using a modified vertex formula, according to the provided preoperative refractive parameters. The size of the implanted ICL V4c was determined based on the white-to-white horizontal corneal diameter and anterior chamber depth.

In the present study, the ICL V4c type (toric or nontoric) and the targeted spherical and cylindrical correction powers were designed as follows. Thirty-one eyes were implanted with toric ICL V4c. Of the 31 eyes, 2 eyes had SE over $-18.00 \mathrm{D}$, in which one eye had cylindrical diopters that have been undercorrected and the other eye had both the cylindrical and the spherical diopters that have been undercorrected; three eyes had preoperative cylindrical diopters from $-4.25 \mathrm{D}$ to $-5.50 \mathrm{D}$, and a cylindrical undercorrection of $-1.25 \mathrm{D}$ to $-1.50 \mathrm{D}$ was designed. Thirty eyes were implanted with nontoric ICL V4c. Of the 30 eyes, 12 eyes had preoperative SE over $-18.00 \mathrm{D}$, in which 3 eyes had cylindrical diopters that have been undercorrected, 1 eye had spherical diopters that have been undercorrected, and 8 eyes had both the cylindrical and the spherical diopters that have been undercorrected; fifteen eyes tried spectacle lenses without cylindrical diopters being corrected before surgery, and the patients were satisfied with the corresponding corrected distance visual acuity, thus chose the nontoric ICL V4c.

ICL V4c implantation procedures were performed by an experienced surgeon (XZ). Pupils were dilated before surgery. After injection of $1 \%$ sodium hyaluronate into the anterior chamber, ICL V4c was then implanted via a $3.0 \mathrm{~mm}$ temporal corneal incision using an injector cartridge and then was placed in the posterior chamber. After that, the viscoelastic surgical agent was washed away using the balanced salt solution, and a miotic agent was instilled. Postoperative medications included antibiotic eye drops, nonsteroidal anti-inflammatory eye drops, steroidal eye drops, and artificial eye drops.

\section{Measurements}

The following parameters were recorded for analysis: uncorrected distance visual acuity (UDVA), corrected distance visual acuity (CDVA), and manifest refractions. The safety index was calculated as the ratio between the CDVA at 2 years and the corresponding CDVA before surgery, and the efficacy index was the ratio between the UDVA at 2 years and the corresponding CDVA before surgery. Intraocular pressure (IOP) was measured with a noncontact tonometer (NCT; Canon, Japan). The central anterior chamber depth (ACD), anterior chamber volume (ACV), anterior chamber angle width (ACAW), and the ICL V4c vault were measured using the Pentacam (Oculus, Germany) through a rotating Scheimpflug camera. The haptic positions of the ICL V4c lens implanted were measured using an ultrasound biomicroscopy (UBM, MD-300L, MEDA Co., Ltd., Tianjin, China). Patient satisfaction was assessed at 2 years. They were asked to rate if they were very satisfied, satisfied, not so satisfied, or very unsatisfied with their visual performance. And overall satisfaction score (ranging from $0=$ very unsatisfied to $10=$ the most satisfied) was also collected.

\section{Statistical Analysis}

Statistical analysis was performed using SAS V9.3 (Cary, NC, USA). For the sake of within-subject correlation, a linear mixed model was used to detect the differences of variables including ACD, ACV, ACAW, IOP, and vault between time points. Correlation analysis was used to evaluate the relationship between two continuous variables. A $P$ value of less than 0.05 was considered statistically significant.

\section{Results}

All surgeries were uneventful, and no vision threatening complications were observed during the follow-up period. 


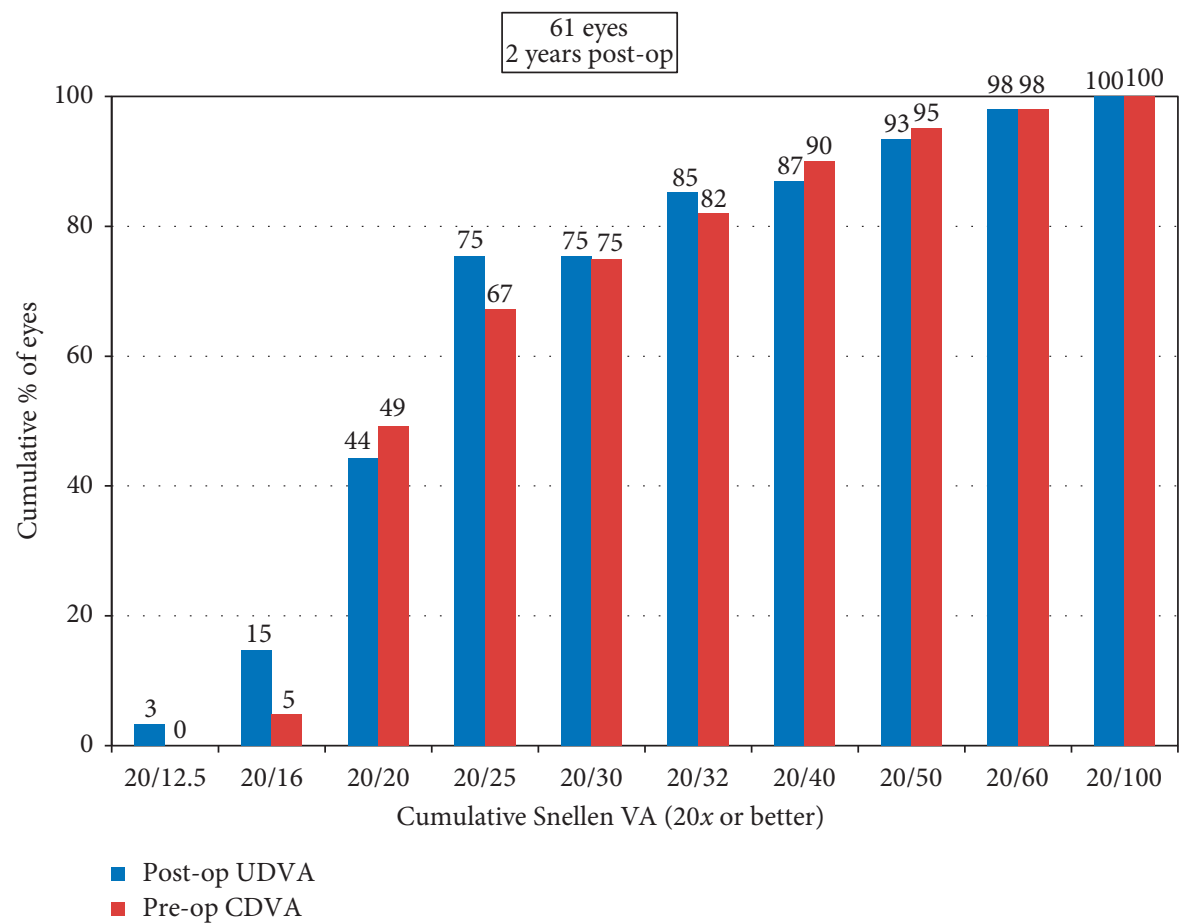

FIGURE 1: Cumulative percentage of the eyes attaining specified cumulative levels of uncorrected distance visual acuity (UDVA) 2 years after surgery.

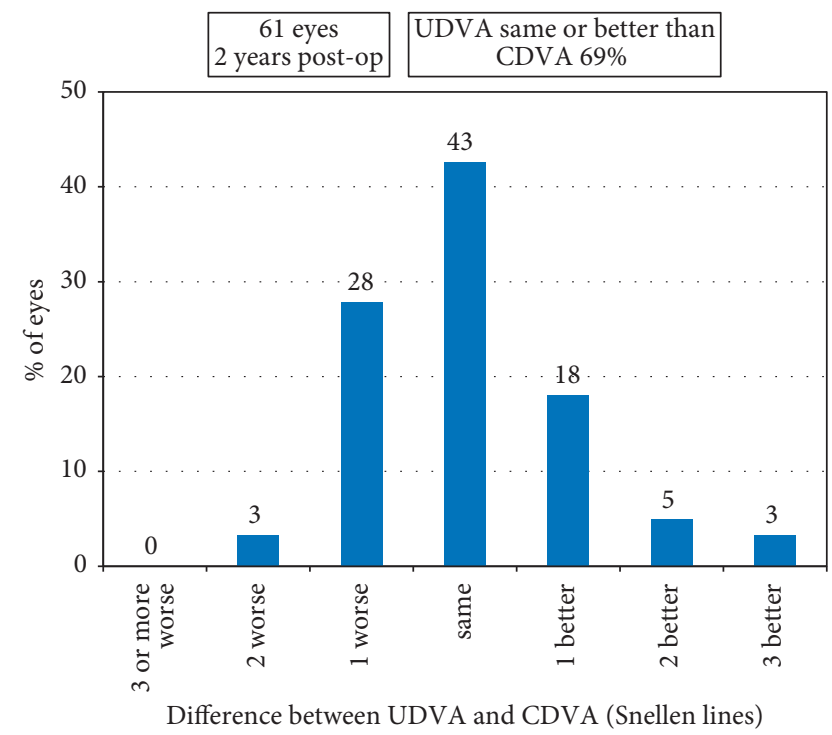

FIGURE 2: Percentage of the eyes comparing 2 years' postoperative uncorrected distance visual acuity (UDVA) and preoperative corrective distance visual acuity (CDVA).

The mean IOP was $15.39 \pm 2.88 \mathrm{mmHg}$ before surgery and $15.86 \pm 4.11 \mathrm{mmHg}$ at 2 years $(p=0.11)$. The mean ECD was $3251 \pm 506 \mathrm{cell} / \mathrm{mm}^{2}$ before surgery and $3246 \pm 522 \mathrm{cell} / \mathrm{mm}^{2}$ at 2 years $(p=0.95)$.

6.1. Refractive Outcomes. The decimal UDVA and CDVA were $0.84 \pm 0.28$ (range, $0.2-1.5$ ) and $1.00 \pm 0.27$ (range, $0.2-1.5)$ at 2 years, respectively. Forty-six of the 61 eyes (75\%) had postoperative UDVA of $20 / 25$ or better

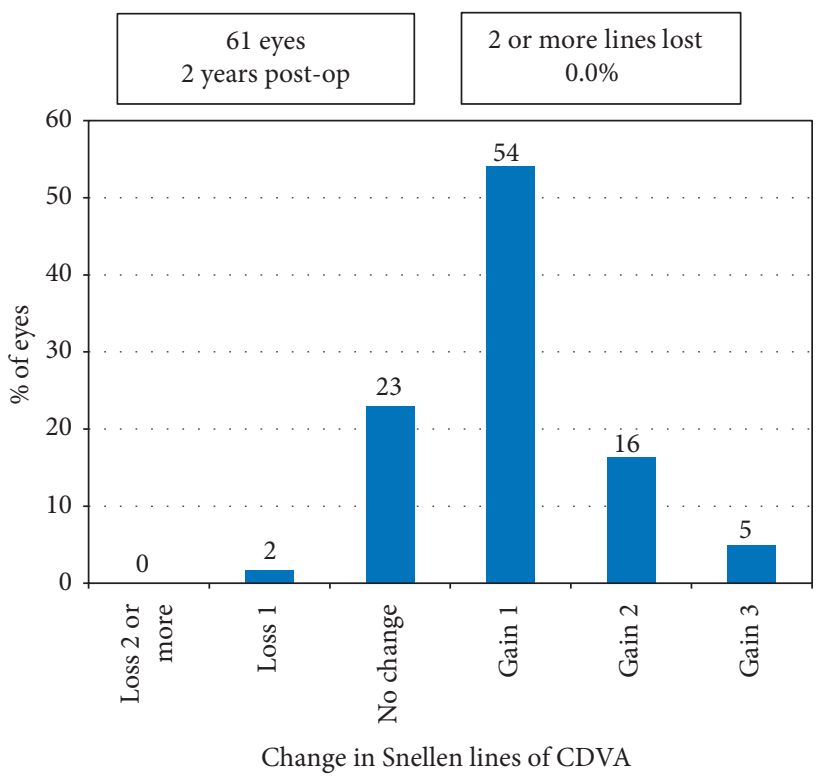

FIGURE 3: Gain and loss of corrected distance visual acuity (CDVA) 2 years postoperatively.

(Figure 1). Forty-two eyes (69\%) had postoperative UDVA same or better than preoperative CDVA (Figure 2). As shown in Figure 3, 14 eyes (23\%) had postoperative CDVA which remained unchanged, 33 eyes (54\%) gained one line, 13 eyes (21\%) gained two or more lines of CDVA, and one eye (2\%) lost one line of CDVA. The efficacy index (the ratio between postoperative UDVA and preoperative CDVA) was $1.03 \pm 0.23$ (range, 0.5 to 1.67 ). The efficacy index was $1.05 \pm$ 0.20 or $1.02 \pm 0.26$ in the eyes with postoperative SE at 2 
years within $\pm 0.50 \mathrm{D}$ or over $-0.50 \mathrm{D}(p=0.65)$. The safety index (the ratio between postoperative CDVA and preoperative CDVA) was $1.24 \pm 0.26$ (range, 0.83 to 2.0 ).

Before operation, 59 eyes $(97 \%)$ had SE over $-9.00 \mathrm{D}$, and 14 eyes $(23 \%)$ had SE over $-18.00 \mathrm{D}$. Mean SE decreased from preoperative $-14.62 \pm 4.29 \mathrm{D}$ to $-0.90 \pm 0.95 \mathrm{D}$ at 2 years $(p<0.0001)$. Mean astigmatism decreased from $-1.82 \pm 1.22 \mathrm{D}$ to $-0.98 \pm 0.93 \mathrm{D}(p<0.001)$. A scatter plot with a best-fit line $\left(y=0.99 x-0.05, R^{2}=0.98\right)$ of the attempted versus the achieved spherical equivalent correction is shown in Figure 4. At 2 years, 79\% (48 eyes) of the eyes were within $\pm 0.50 \mathrm{D}$ and $98 \%$ (60 eyes) were within $\pm 1.00 \mathrm{D}$ of the intended correction (Figure 5). Before operation, the percentage of the eyes with astigmatism $\leq 0.50 \mathrm{D}$, $\leq 1.00 \mathrm{D}, \leq 2.00 \mathrm{D}$, and $\leq 3.00 \mathrm{D}$ were $11.5 \%, 26 \%, 72 \%$, and $90 \%$, respectively, and at 2 years, they increased to $44 \%, 67 \%$, $92 \%$, and $97 \%$, respectively (Figure 6). For the 31 eyes implanted with toric ICL V4c, a scatter plot with a best-fit line $\left(y=0.94 x-0.01, R^{2}=0.92\right)$ of the target versus the surgically induced astigmatism is shown in Figure 7.

All patients were satisfied with their visual performance, and $71 \%$ were more than satisfied (very satisfied). The mean score of overall satisfaction was $9.27 \pm 0.87$ (range, 8 to 10). And all the patients chose "yes," when asked "Would you consider recommending this operation to patients like you?"

6.2. Anterior Chamber Changes. Mean ACV was $193.28 \pm$ $29.15 \mathrm{~mm}^{3}$, mean ACD was $3.15 \pm 0.23 \mathrm{~mm}$, and mean ACAW was $36.51 \pm 6.54$ degree before operation; they, respectively, decreased to $112.48 \pm 17.01 \mathrm{~mm}^{3}, 2.99 \pm 0.23 \mathrm{~mm}$, and $22.54 \pm$ 5.27 degree at 2 years ( $p=0.0008,0.008$, and 0.0003 , resp.). Figure 8 showed an example of anterior chamber changes measured by Pentacam before operation and at 2 years postoperatively.

The postoperative ACD was significantly correlated with preoperative ACD (Spearman's rho, $r=0.85, p<0.0001$ ). The postoperative ACV was significantly correlated with preoperative ACV $(r=0.60, p<0.0001)$. No significant relationship was found between postoperative and preoperative ACAW $(r=0.21, p=0.10)$. IOP at 2 years was not significantly correlated with postoperative ACD $(r=-0.04$, $p=0.76)$, ACV $(r=0.09, p=0.50)$, or ACAW $(r=0.18$, $p=0.17$.

Mean vault at 1 day, 1 week, and 2 years was $426 \pm$ $168 \mu \mathrm{m}, 463 \pm 186 \mu \mathrm{m}$, and $449 \pm 167 \mu \mathrm{m}$, respectively $(p=0.72)$. Correlation analyses revealed that vault at 2 years was significantly correlated with postoperative ACAW $(r=-0.67, p<0.0001)$ and ACV $(r=-0.36, p=0.004)$ but was of no significant relationship with ACD $(r=0.20$, $p=0.13)$.

6.3. ICL V4c Haptics Positions. The positions of the ICL V4c haptics were examined in 46 eyes ( 24 patients). Figure 9 showed examples of haptic positions at 2 years after surgery. Six percent of the eyes $(3 / 46)$ had four haptics located in the ciliary sulcus (Figure 9(a)), 70\% of the eyes (32/46) had four haptics located in the ciliary processes (Figure 9(b)), and
$24 \%$ eyes $(11 / 46)$ had haptics located either in the ciliary processes or in the ciliary sulcus.

\section{Discussion}

The present study reported 2-year results after the ICL V4c implantation in a group of high-myopic patients. Refractive outcomes, anterior chamber changes, and haptic position in the posterior chamber were evaluated, which could help to better understand the latest generation of ICL.

The efficacy and safety indexes of 1.03 and 1.24 in this study are comparable with previous studies; for example, the indexes were 1.00 and 1.01 at 6 months in Alfonso's study [3], 1.03 and 1.13 at 6 months in Shimizu's study [2], and 1.00 and 1.04 at 12 months in Lisa's study [4]. Compared with the above studies, the mean postoperative SE was more myopic in the current study. The following reasons are listed. Firstly, this study population was mostly consisted of high myopic (SE of $-8.00 \mathrm{D}$ or higher) adults. The mean preoperative SE was $-14.62 \mathrm{D}$, extremely higher than the abovementioned studies with the mean SE of $-7.30 \mathrm{D}$ to $-8.80 \mathrm{D}[2-4]$. Owing to the high percentage of super-high myopia $(23 \%$ over $-18.00 \mathrm{D})$ in the study group, the residual myopic diopters existed for they were beyond the ICL V4c correction range. Secondly, the mean postoperative astigmatism was also higher in this study, and some of the eyes had astigmatism undercorrected for different reasons as we described in Patients and Methods, which lead to the existence of residual cylindrical diopters in our study. Still and all, the predictability results showed that $98 \%$ of the eyes had their attempted SE within $\pm 1.00 \mathrm{D}$ of the intended correction. Therefore, despite the residual refractive diopters, the correction efficiency is satisfying. The extremely high diopters were significantly decreased with no vision threatening complications occurred; $75 \%$ had UDVA of 20/25 or higher, and $75 \%$ of the eyes gained more lines of CDVA after operation, suggesting efficacy and safety of ICL V4c implantation for correcting super-high myopia.

In another study, we found that ICL implantation is safe and effective for the correction of residual refractive error after corneal refractive surgeries [5]. Conversely, if the cornea condition fits the operation indications, corneal laser surgeries would be suitable to treat the residual diopters after ICL implantation. Sánchez-Galeana et al. reported that LASIK or PRK can be used to treat the residual refractive error following posterior chamber phakic IOL implantation [6]. Zaldivar et al. reported that combined posterior chamber phakic IOL implantation with LASIK was an effective and predictable method for correcting myopia from -18 to -35 D [7]. Comparative studies found no significant difference between the outcomes of the conventional ICL and the hole ICL [8-10]. The combination of ICL V4c with corneal refractive surgery to treat super-high myopia could be further explored.

The most common concerns regarding ICL implantation are cataract formation and secondary glaucoma [11]. None of the eyes in the present study developed cataract, and no IOP increase was observed at 2 years. The results are consisting with previous studies [2-4]. A few studies reported 


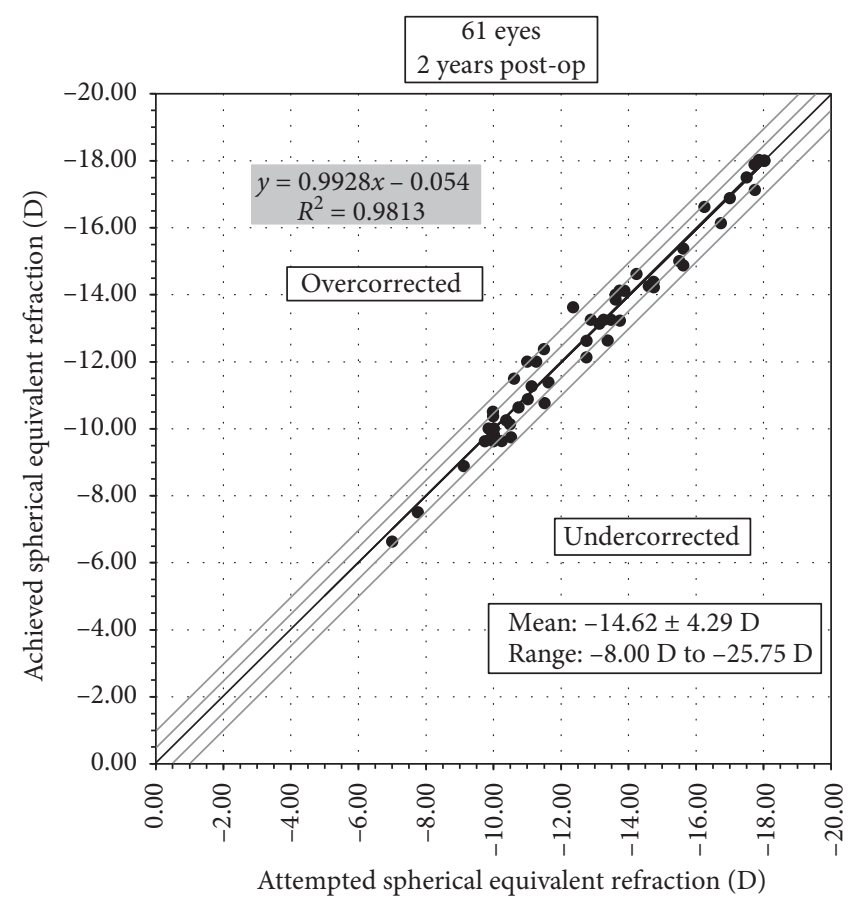

Figure 4: Attempted spherical equivalent refraction change versus the achieved spherical equivalent refraction change at 2 years.

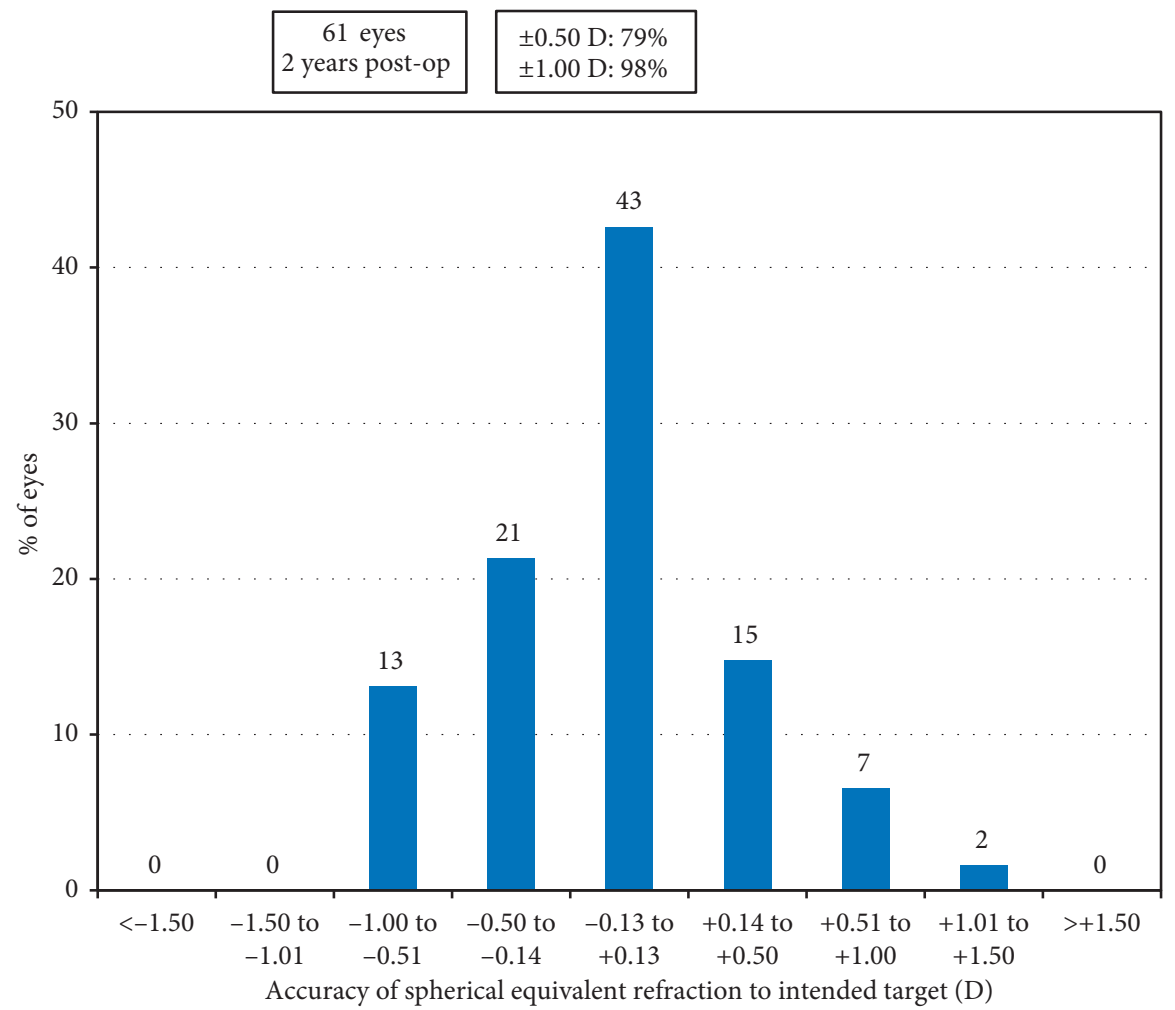

Figure 5: Percentage of the eyes attaining specified differences in attempted versus achieved correction at 2 years.

that anterior subcapsular opacification rather than clinically significant cataract might happen after ICL V4c implantation, and the rate (approximately $3 \%$ ) was half of that in $\mathrm{V} 4 \mathrm{~b}$ $[12,13]$. The insertion of an ICL will reduce the aqueous humor circulation and cause a metabolic disturbance of the crystalline lens [14]. The innovative central hole design of ICL V4c increases aqueous humor flow onto the crystalline lens, potentially decreases the risk of cataract formation after ICL V4c implantation [15]. Sanders estimated through Kaplan-Meier analyses that about $7 \%$ of the eyes develop 


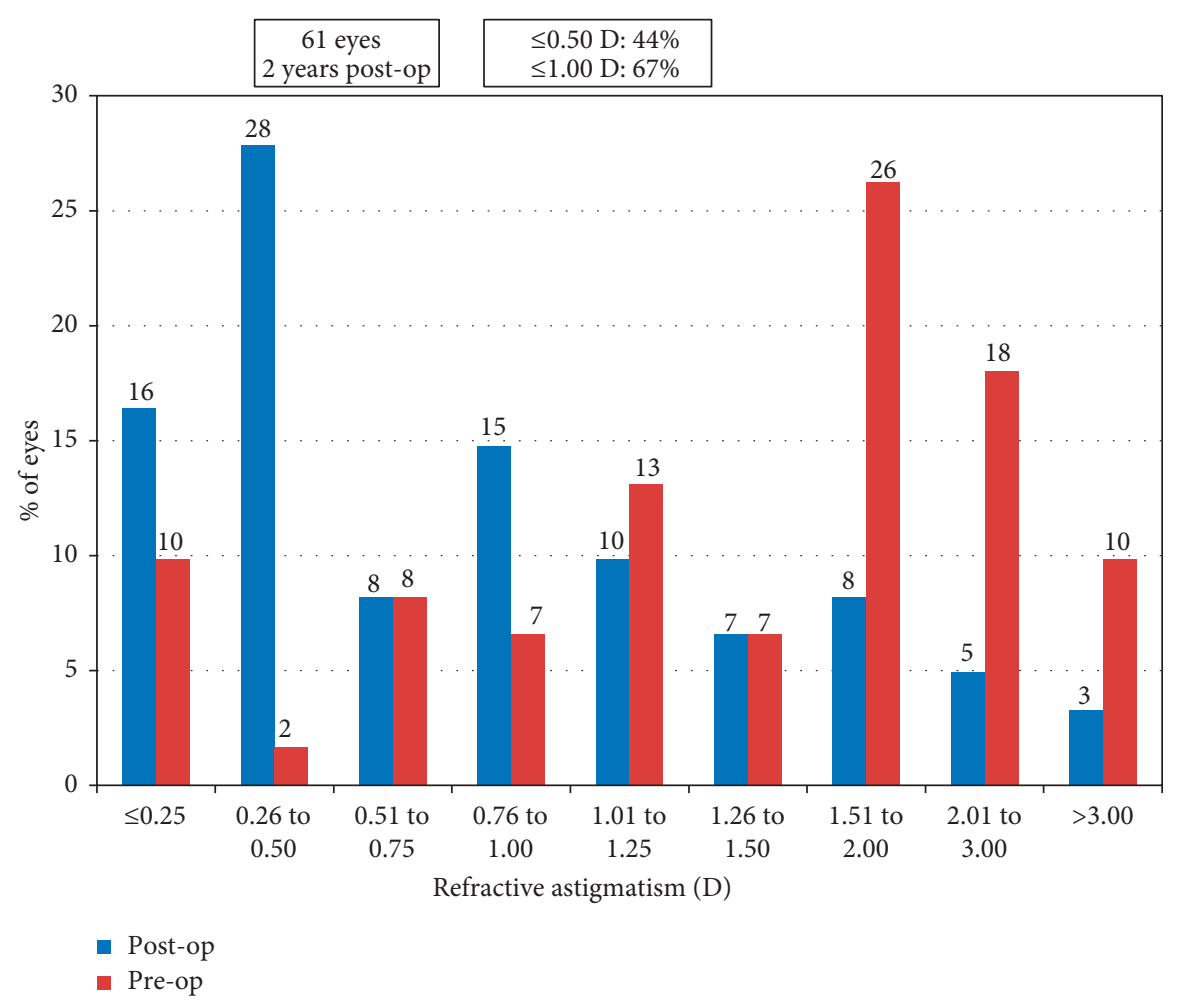

FIGURE 6: Percentage of the eyes within different astigmatism ranges before operation and at 2 years.

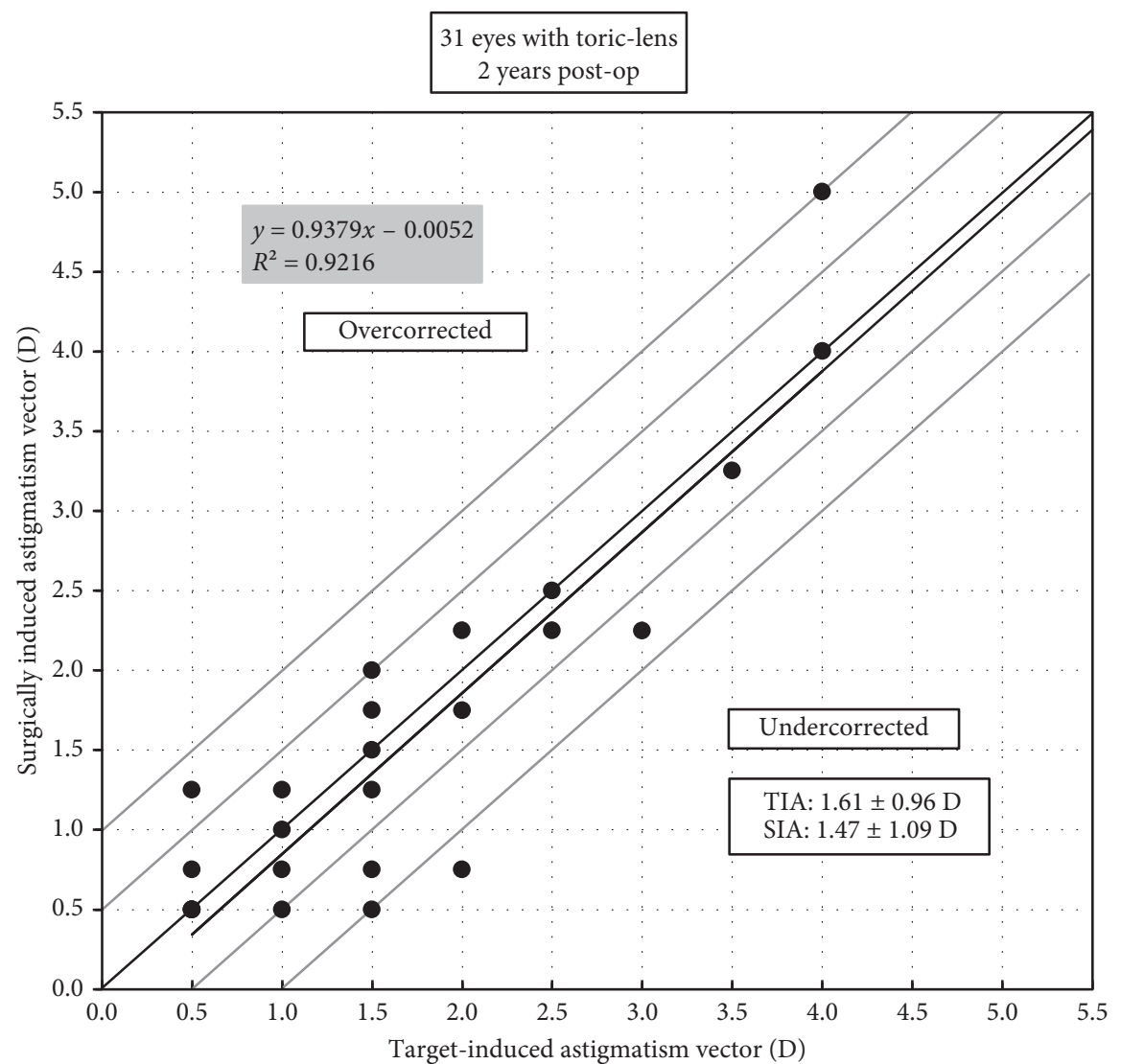

FIGURE 7: Target-induced astigmatism (TIA) versus surgically induced astigmatism (SIA) at 2 years in the eyes implanted with toric ICL V4c. 


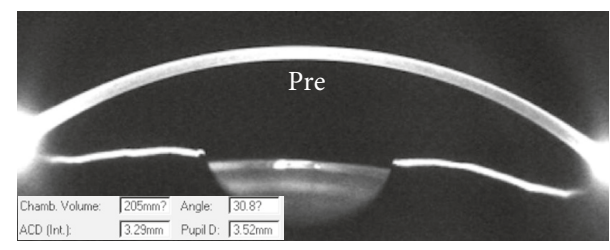

(a)

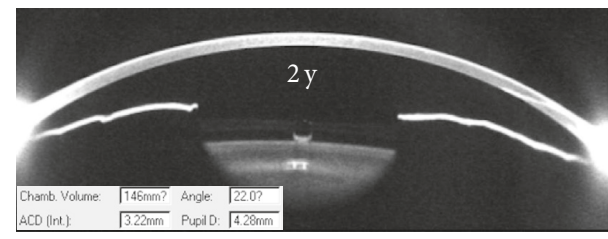

(b)

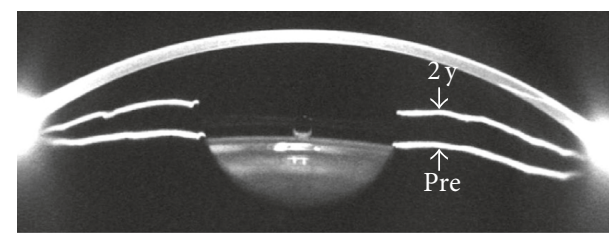

(c)

Figure 8: Pentacam sample images of the anterior chamber segment changes before and 2 years after the ICL V4c was implanted. Patient information: male, 31 years, left eye, with preoperative spherical equivalent refraction of $-10.88 \mathrm{D}\left(-10.50 \mathrm{DS} /-0.75 \mathrm{DC} \times 130^{\circ}\right)$, and a nontoric ICL V4c was implanted. Before surgery, the anterior chamber volume was $205 \mathrm{~mm}^{3}$, the central anterior chamber depth was $3.29 \mathrm{~mm}$, and the anterior chamber angle was 30.8 degree (a). At 2 years, the anterior chamber volume was $146 \mathrm{~mm}^{3}$, the central anterior chamber depth was $3.22 \mathrm{~mm}$, the anterior chamber angle was 22.0 degree, and the vault was $470 \mu \mathrm{m}$ (b). The merged image (c) of A and B showed that the iris was pushed forward after ICL V4c implantation.

anterior subcapsular opacification at 7 years after ICL implantation, and only $2 \%$ will progress to visually significant cataracts [16]. Longer-term complications after ICL V4c implantation need to be further observed.

Vault was defined as the central distance between the posterior surface of the ICL V4c and the anterior surface of the crystalline lens. Excessive low vault increases the risk of cataract formation for the artificial lens might touch the crystalline lens. On the contrast, extremely high vault might induce angle closure glaucoma. In Gonvers's study regarding $\mathrm{V} 3$ and V4, they suggested that a vault greater than $150 \mu \mathrm{m}$ (at least $90 \mu \mathrm{m}$ ) was able to avoid the lens contact and prevented cataract formation [17]. The vault was pretty stable between time points in this study, ranging from $130 \mu \mathrm{m}$ to $810 \mu \mathrm{m}$ at 2 years. There is one eye in our study that had the vault $(130 \mu \mathrm{m})$ lower than $150 \mu \mathrm{m}$ but higher than $90 \mu \mathrm{m}$ and currently without developing the anterior subcapsular opacification. Pupil size/movement, crystalline lens thickness changes, and the ICL haptics position were considered to be influential factors of vault [18-20]. We found in this study group that the higher the vault, the narrower the anterior chamber angle width, and the smaller the anterior chamber volume at postoperative 2 years. A recent study also reported a weak relationship between the anterior chamber angle width and vault at 18 months after

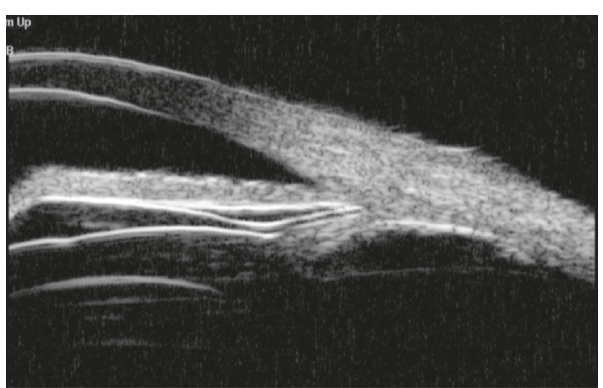

(a)

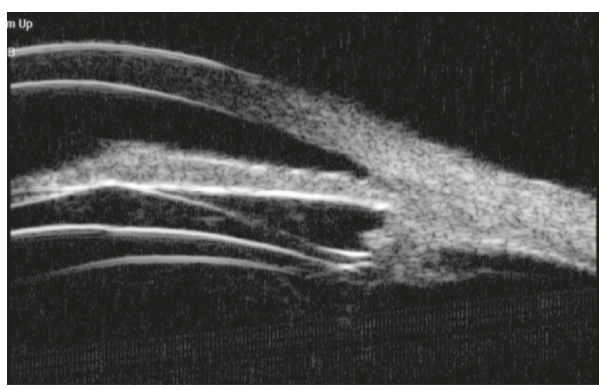

(b)

FIGURE 9: Ultrasound biomicroscopy sample images of the ICL V4c haptic position and its relationship with the ciliary body. The haptics were either located in the ciliary sulcus (a) or in the ciliary processes (b).

ICL V4c implantation [21]. It is obvious that the higher the vault, the more the implanted lens pushes forward the iris to the cornea, the more the reduction of the anterior chamber space, and therefore, the more the attention should be paid to the intraocular pressure changes.

Anterior chamber segment changes after ICL implantation were evaluated using different methods, such as Fourier-domain optical coherence tomography (FD-OCT) [22] and UBM [23]. We used Pentacam in the current study and found that the anterior chamber angle width decreased to $62 \%$ at 2 years. Eissa et al. also found that angle width in degree decreased to $64 \%$ of the preoperative value at 18 month follow-up time point using the Scheimpflug tomography imaging system, from preoperative 40.14 degree to 25.49 degree [21]. Similarly, no correlation was found between anterior chamber angle width and IOP after operation in their study. The narrowing of anterior chamber angle after ICL implantation usually achieves stability in 1 month after ICL implantation. For example, in 48 eyes of 29 patients with high myopia treated with the traditional ICL, Chung et al. reported that trabecular-iris angle decreased by $42 \%$ at 1 month, and no significant progressive changes were observed thereafter over a mean follow-up of 33 months [23]. Fernández-Vigo et al. reported that trabecular-iris angle measured with FD-OCT reduced by $34 \%$ to $42 \%$ at 1 month after ICL V4c implantation and then remained stable at 3 months [22]. The above evidences suggested that the narrowing of the anterior chamber angle after ICL V4c implantation had little impact on intraocular pressure, and the stable status could be achieved in 1 month. A carefully monitoring of anterior chamber angle and IOP is required in 
the early postoperative period. However, despite that the longer period results suggested a great stability of anterior chamber angle, regular visits are essential as the anterior chamber space decreases with age, especially after 40 years of age [24].

The abnormal position of ICL in the posterior chamber is the main cause of cataract and glaucoma $[16,25]$. Ultrasound biomicroscopy (UBM) is a unique way to noninvasively evaluate the position of ICL in the posterior chamber and its relation with adjacent tissues, such as the ciliary body, crystalline lens, and iris. There were very few studies which evaluated the haptics position of ICL V4c in the posterior chamber. A recent study reported that the haptics of V4 was located in the ciliary sulcus in $112(79 \%)$ of their 142 eyes at 6 months, and at least one haptic was found to locate on the lens periphery and zonules in $21 \%$ of the eyes [26]. The haptics of ICL V4c were mostly located in the ciliary processes in our study and did not increase the risk of anterior subcapsular opacification or high IOP. And the vault was stable during the 2 years, indicating a stable position of the ICL V4c lens.

In conclusion, implantation of ICL V4c is an effective, safe, and predictive option for correcting high and superhigh myopia. Pentacam and UBM can provide detailed anatomical information to monitor the ICL V4c position and its relationship with adjacent tissues. The decreased anterior chamber space after ICL V4c implantation did not induce intraocular pressure increasing during the 2-year follow-up.

\section{Data Availability}

The data could be found in the Department of Ophthalmology, Eye and ENT Hospital of Fudan University, Myopia Key Laboratory of the Health Ministry, Shanghai.

\section{Conflicts of Interest}

The authors declare that they have no conflicts of interest.

\section{Authors' Contributions}

Drs. Zhipeng Yan and Huamao Miao contributed equally to this work and should be considered as equal first authors.

\section{Acknowledgments}

This study was supported by the Natural Science Foundation of China for Young Scholars (Grant nos. 81700872 and 81500753), the Shanghai Shenkang Hospital Development Center (Grant no. SHDC12016207), the National Natural Science Foundation of China (Grant nos. 81770955 and 81570879) and the Project of Shanghai Science and Technology (Grant nos. 17140902900 and 17411950200).

\section{References}

[1] J. Sun, J. Zhou, P. Zhao et al., "High prevalence of myopia and high myopia in 5060 Chinese university students in
Shanghai," Investigative Opthalmology and Visual Science, vol. 53, no. 12, pp. 7504-7509, 2012.

[2] K. Shimizu, K. Kamiya, A. Igarashi, and T. Shiratani, "Early clinical outcomes of implantation of posterior chamber phakic intraocular lens with a central hole (Hole ICL) for moderate to high myopia," British Journal of Ophthalmology, vol. 96, no. 3, pp. 409-412, 2012.

[3] J. F. Alfonso, C. Lisa, L Fernandez-Vega Cueto, L. BeldaSalmeron, D. Madrid-Costa, and R. Montes-Mico, "Clinical outcomes after implantation of a posterior chamber collagen copolymer phakic intraocular lens with a central hole for myopic correction," Journal of Cataract and Refractive Surgery, vol. 39, no. 6, pp. 915-921, 2013.

[4] C. Lisa, M. Naveiras, B. Alfonso-Bartolozzi, L. Belda-Salmeron, R. Montes-Mico, and J. F. Alfonso, "Posterior chamber collagen copolymer phakic intraocular lens with a central hole to correct myopia: one-year follow-up," Journal of Cataract and Refractive Surgery, vol. 41, no. 6, pp. 1153-1159, 2015.

[5] X. Chen, X. Y. Wang, X. Zhang, Z. Chen, and X. T. Zhou, "Implantable collamer lens for residual refractive error after corneal refractive surgery," International Journal of Ophthalmology, vol. 9, no. 10, pp. 1421-1426, 2016.

[6] C. A. Sanchez-Galeana, R. J. Smith, X. Rodriguez, M. Montes, and A. S. Chayet, "Laser in situ keratomileusis and photorefractive keratectomy for residual refractive error after phakic intraocular lens implantation," Journal of Refractive Surgery, vol. 17, no. 3, pp. 299-304, 2001.

[7] R. Zaldivar, J. M. Davidorf, S. Oscherow, G. Ricur, and V. Piezzi, "Combined posterior chamber phakic intraocular lens and laser in situ keratomileusis: bioptics for extreme myopia," Journal of Refractive Surgery, vol. 15, no. 3, pp. 299-308, 1999.

[8] T. Huseynova, S. Ozaki, T. Ishizuka, M. Mita, and M. Tomita, "Comparative study of 2 types of implantable collamer lenses, 1 with and 1 without a central artificial hole," American Journal of Ophthalmology, vol. 157, no. 6, pp. 1136-1143, 2014.

[9] K. Kamiya, K. Shimizu, A. Saito, A. Igarashi, and H. Kobashi, "Comparison of optical quality and intraocular scattering after posterior chamber phakic intraocular lens with and without a central hole (hole ICL and conventional ICL) implantation using the double-pass instrument," PLoS One, vol. 8, no. 6, Article ID e66846, 2013.

[10] K. Shimizu, K. Kamiya, A. Igarashi, and H. Kobashi, "Longterm comparison of posterior chamber phakic intraocular lens with and without a central hole (hole ICL and conventional ICL) implantation for moderate to high myopia and myopic astigmatism: consort-compliant article," Medicine, vol. 95, no. 14, article e3270, 2016.

[11] J. F. Alfonso, C. Lisa, A. Abdelhamid, P. Fernandes, J. Jorge, and R. Montes-Mico, "Three-year follow-up of subjective vault following myopic implantable collamer lens implantation," Graefe's Archive for Clinical and Experimental Ophthalmology, vol. 248, no. 12, pp. 1827-1835, 2010.

[12] V. Bhandari, S. Karandikar, J. K. Reddy, and K. Relekar, "Implantable collamer lens V4b and V4c for correction of high myopia," Journal of Current Ophthalmology, vol. 27, no. 3-4, pp. 76-81, 2015.

[13] S. Karandikar, V. Bhandari, and J. Reddy, "Outcomes of implantable collamer lens V4 and V4c for correction of high myopia-a case series," Nepalese Journal of Ophthalmology, vol. 7, no. 2, pp. 164-172, 2015.

[14] K. Fujisawa, K. Shimizu, S. Uga et al., "Changes in the crystalline lens resulting from insertion of a phakic IOL (ICL) into the porcine eye," Graefe's Archive for Clinical and Experimental Ophthalmology, vol. 245, no. 1, pp. 114-122, 2007. 
[15] T. Shiratani, K. Shimizu, K. Fujisawa, S. Uga, K. Nagano, and Y. Murakami, "Crystalline lens changes in porcine eyes with implanted phakic IOL (ICL) with a central hole," Graefe's Archive for Clinical and Experimental Ophthalmology, vol. 246, no. 5, pp. 719-728, 2008.

[16] D. R. Sanders, "Anterior subcapsular opacities and cataracts 5 years after surgery in the Visian Implantable Collamer Lens FDA Trial," Journal of Refractive Surgery, vol. 24, no. 6, pp. 566-570, 2008.

[17] M. Gonvers, C. Bornet, and P. Othenin-Girard, "Implantable contact lens for moderate to high myopia: relationship of vaulting to cataract formation," Journal of Cataract and Refractive Surgery, vol. 29, no. 5, pp. 918-924, 2003.

[18] K. Kamiya, K. Shimizu, and M. Komatsu, "Factors affecting vaulting after implantable collamer lens implantation," Journal of Refractive Surgery, vol. 25, no. 3, pp. 259-264, 2009.

[19] K. Kamiya, K. Shimizu, and T. Kawamorita, "Changes in vaulting and the effect on refraction after phakic posterior chamber intraocular lens implantation," Journal of Cataract and Refractive Surgery, vol. 35, no. 9, pp. 1582-1586, 2009.

[20] X. Chen, H. Miao, R. K. Naidu, X. Wang, and X. Zhou, "Comparison of early changes in and factors affecting vault following posterior chamber phakic Implantable Collamer Lens implantation without and with a central hole (ICL V4 and ICL V4c)," BMC Ophthalmology, vol. 16, no. 1, p. 161, 2016.

[21] S. A. Eissa, S. H. Sadek, and M. W. El-Deeb, "Anterior chamber angle evaluation following phakic posterior chamber collamer lens with centraflow and its correlation with ICL vault and intraocular pressure," Journal of Ophthalmology, vol. 2016, Article ID 1383289, 7 pages, 2016.

[22] J. I. Fernandez-Vigo, A. Macarro-Merino, C. Fernandez-Vigo et al., "Effects of Implantable Collamer Lens V4c Placement on Iridocorneal Angle Measurements by Fourier-Domain Optical Coherence Tomography," American Journal of Ophthalmology, vol. 162, pp. 43.e1-52.e1, 2016.

[23] T. Y. Chung, S. C. Park, M. O. Lee, K. Ahn, and E. S. Chung, "Changes in iridocorneal angle structure and trabecular pigmentation with STAAR implantable collamer lens during 2 years," Journal of Refractive Surgery, vol. 25, no. 3, pp. 403409, 2009.

[24] Y. Zong, Q. Xu, C. Jiang, H. Zhu, J. Yu, and X. Sun, "Measurement of and factors associated with the anterior chamber volume in healthy Chinese adults," Journal of Ophthalmology, vol. 2017, Article ID 6762047, 6 pages, 2017.

[25] J. S. Chang and A. Y. Meau, "Visian Collamer phakic intraocular lens in high myopic Asian eyes," Journal of Refractive Surgery, vol. 23, no. 1, pp. 17-25, 2007.

[26] A. M. Elshafei, M. M. Genaidy, and H. M. Moharram, "Vivo positional analysis of implantable collamer lens using ultrasound biomicroscopy," Journal of Ophthalmology, vol. 2016, Article ID 4060467, 5 pages, 2016. 


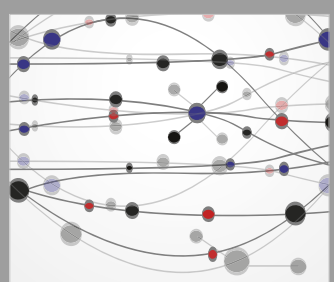

The Scientific World Journal
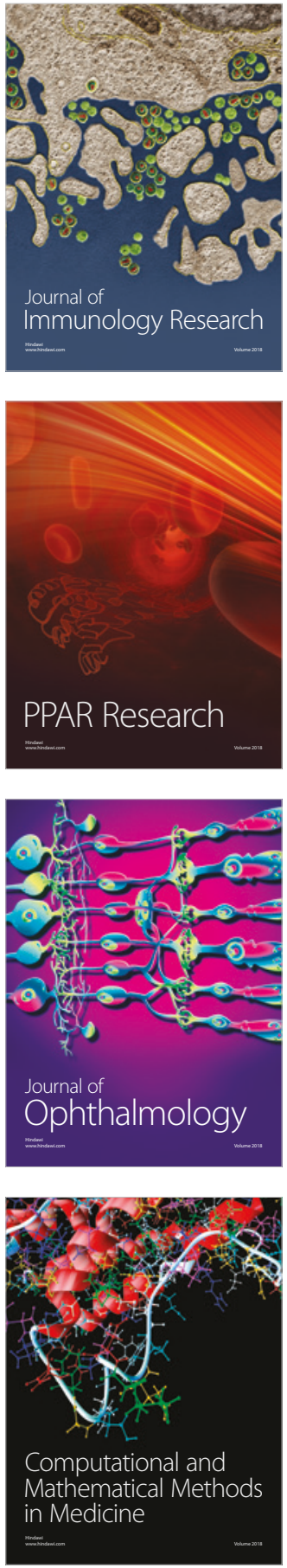

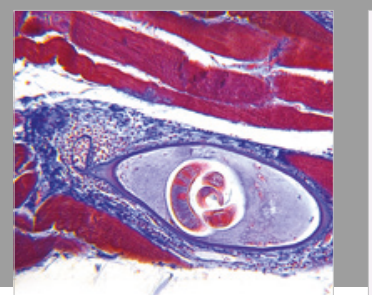

Gastroenterology Research and Practice

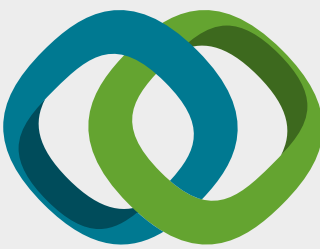

\section{Hindawi}

Submit your manuscripts at

www.hindawi.com
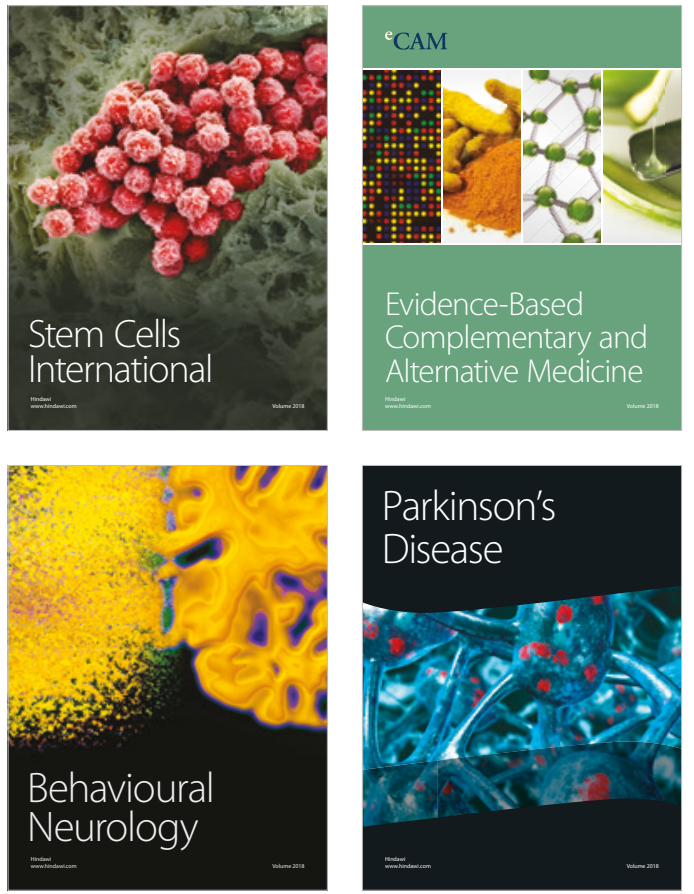

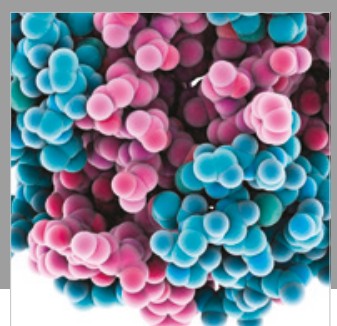

ournal of

Diabetes Research

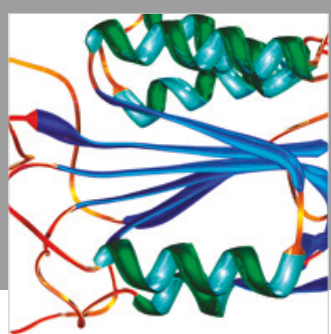

Disease Markers
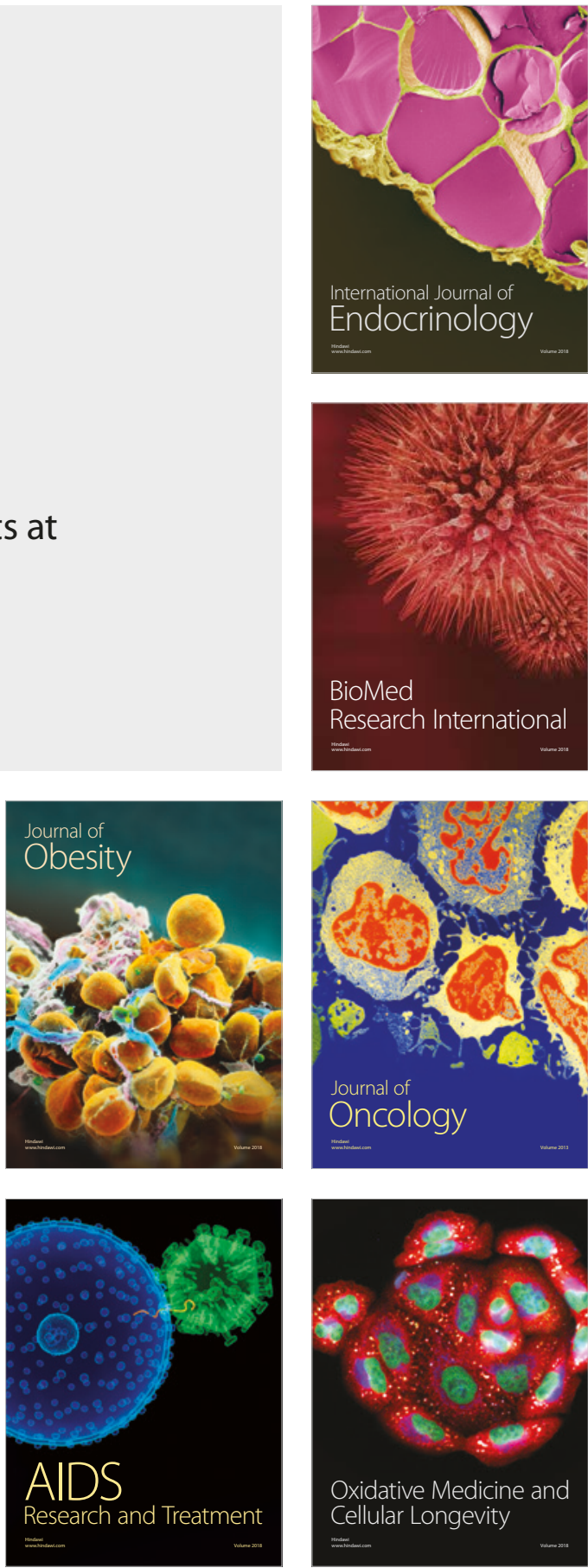